\title{
Korean First Prospective Phase II Study, Feasibility of Prone Position in Postoperative Whole Breast Radiotherapy: A Dosimetric Comparison
}

\author{
Yoonsun Chung, $\mathrm{PhD}^{1}$ \\ Jeong II $\mathbf{Y u}, \mathrm{MD}, \mathrm{PhD}{ }^{2}$ \\ Won Park, MD, PhD² \\ Doo Ho Choi, MD, PhD²
}

${ }^{1}$ Department of Nuclear Engineering,

Hanyang University, Seoul, ${ }^{2}$ Department of Radiation Oncology, Samsung Medical Center, Sungkyunkwan University School of Medicine, Seoul, Korea

\begin{abstract}
Purpose
This first Korean prospective study is to evaluate the feasibility of prone breast radiotherapy after breast conserving surgery for left breast cancer patients who have relatively small breast size and we present dosimetric comparison between prone and supine positions.
\end{abstract}

\section{Materials and Methods}

Fifty patients underwent two computed tomography (CT) simulations in supine and prone positions. Whole breast, ipsilateral lung, heart, and left-anterior-descending coronary artery were contoured on each simulation CT images. Tangential-fields treatment plan in each position was designed with total 50 Gy in 2-Gy fractions, and then one of the positions was designated for the treatment by comparing target coverage and dose to normal organs. Also, interfractional and intrafractional motion was evaluated using portal images.

\section{Results}

In total 50 patients, 32 cases were decided as prone-position-beneficial group and 18 cases as supine-position-beneficial group based on dosimetric advantage. Target dose homogeneity was comparable, but target conformity in prone position was closer to optimal than in supine position. For both group, prone position significantly increased lung volume. However, heart volume was decreased by prone position for prone-position-beneficial group but was comparable between two positions for supine-position-beneficial group. Lung and heart doses were significantly decreased by prone position for prone-position-beneficial group. However, prone position for supine-position-beneficial group increased heart dose while decreasing lung dose. Prone position showed larger interfractional motion but smaller intra-fractional motion than supine position.

\section{Conclusion}

Prone breast radiotherapy could be beneficial to a subset of small breast patients since it substantially spared normal organs while achieving adequate target coverage.

\section{Key words}

Clinical trial, Left-sided breast neoplasms, Radiotherapy,

Prone position

\section{Introduction}

Breast cancer is the most common cancer in women worldwide [1] and the second most common cancer in Korean women [2]. While postoperative whole breast radiotherapy (RT) has previously been proven effective in clinical trials [3], the concern about cardiac problems according to exposed dose has been raised especially in left-sided breast cancer patients. Meta-analysis of U.S. breast cancer RT between 1973 and 2001 found an increase in heart-related mortality, when older equipment and techniques had yet been utilized [4]. The reduction of heart perfusion according to RT volume was also reported to be induced in $40 \%$ of patients within 2 years [5]. In addition, we also see the coronary arteries damaged by postoperative RT in left breast cancer patients compared to the right [6]. A paper published in the New England Journal of Medicine [7] reported that main coronary artery 
disease increased by $7.4 \%$ per $1 \mathrm{~Gy}$ of radiation exposure to the heart (95\% confidence interval, 2.9 to $14.5 \%$ ), and there was no threshold dose. Furthermore, this heart disease begins within 5 years after RT and appears to be continued up to 30 years [7]. Even though recent paper [8] concluded that study based on large population receiving whole breast RT between 1990 and 1999 with median follow-up period of 15.5 years revealed no increase of cardiac-related mortality to left breast cancer patients, cardiac dose is still important consideration for whole breast RT.

Besides heart, pulmonary toxicity is also one of the problems which cannot be ignored in long-term survivors who received postoperative breast $\mathrm{RT}$. Although the incidence of clinical radiation pneumonitis after breast RT is not high, radiologic changes in lung due to the radiation have been observed in $20 \%-40 \%$ of the patients [9]. These radiation exposures have been reported to cause lung function deterioration in long-term survivors [10]. Therefore, although the incidence of pneumonia is low after RT, it is necessary to reduce the radiation dose to lungs to preserve pulmonary function in long-term survivors.

Breast cancer patients have received RT in supine position with autonomic respiration, which is superior in terms of patient comfort and reproducibility. The above-mentioned studies have also been conducted in supine position with autonomic respiration. There have been attempts to reduce the radiation dose to the heart and lungs in postoperative whole breast RT by applying prone or lateral decubitus positions and respiratory motion management [11-15]. Since prone position was considered to be useful only in patients with large volume of breast $(>1,000 \mathrm{~mL})$, most of them were studied mainly in United States and Europe. It was repeatedly shown that RT in prone position can reduce cardiac radiation exposure in left breast cancer [16-21]. Recently, it has been reported that radiation dose to heart or lung is reduced by prone position for relatively smaller breast size $[22,23]$. The results of this study gave the motivation that it is possible to reduce radiation dose to heart and lungs by means of the use of prone position even in Korean patients whose average breast volume is much smaller. However, in Korea, postoperative RT is performed in supine position for most of the cases, and respiratory motion management RT has been tried in some institutions [24].

Yet, the effectiveness of prone position in breast RT has not been reported for Korean women with relatively small breast. Hence, we proposed the first Korean prospective phase II study to evaluate the feasibility of prone position in the whole breast RT for Korean left breast cancer patients.

\section{Materials and Methods}

\section{Patients}

Fifty left breast cancer female patients receiving whole breast irradiation after breast conserving surgery participated in this study and provided written informed consent from December 2014 to June 2015. The inclusion criteria of this study were as follows: age between 20 and 70 years with pathologically confirmed left-sided breast cancer after curative resection and Eastern Cooperative Oncology Group performance status 0 or 1 . We excluded patient who needs the irradiation of loco-regional lymph nodes area, who has combined distant metastasis, who is pregnant or breast-feeding, who has previous RT history of chest or neck area, or who is not indicated postoperative whole breast RT. Based on the results of previous clinical studies about prone breast RT, we could hypothesize that the prone position can reduce $50 \%$ of heart irradiation dose in $80 \%$ of left breast cancer patients compared to the supine position. A total number of 50 patients were calculated to verify this hypothesize at an alpha of 0.05 , a power of $80 \%$, and dropout rate of $10 \%$.

\section{Computed tomography simulations}

Each patient underwent two computed tomography (CT) simulations for whole breast irradiation: the first in supine position and the second in prone position. First, CT images were obtained in conventional supine and arm-up position on a breast board (CIVCO Medical Solutions, Kalona, IA). After that, second CT images were acquired in prone position using a custom-made prone breast board made of polyurethane foam with an aperture positioning left breast and a wedge to set contralateral breast away from the RT fields. Patient's face was turned to the left to limit the rotation of her body in prone position (Fig. 1). For both setups, radioopaque wires were placed to indicate palpable breast mass and midline of chest of the patients. On the prone breast board, radio-opaque markers were attached to point out the position of aperture and the top of board in order to provide accurate information for RT plan. Both CT images were obtained in 3.75-mm thick slices without contrast, and then transferred to the treatment planning system, Pinnacle3 (Philips Healthcare, Fitchburg, WI).

\section{RT planning and dosimetric comparison}

The whole breast as a clinical target volume (CTV), ipsilateral lung, heart, and left anterior descending coronary artery (LADCA) were contoured on each CT images by a radiation oncologist according to the Radiation Therapy Oncology 


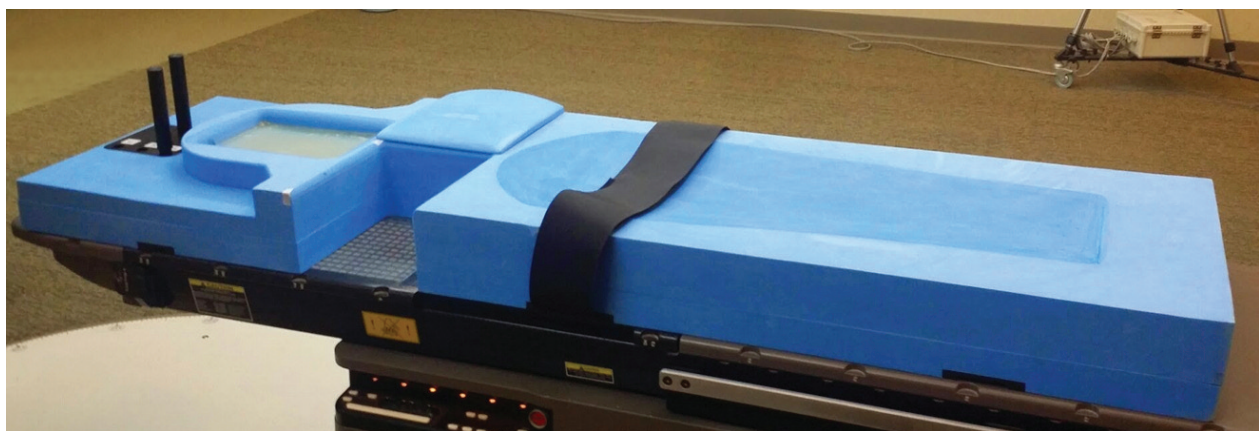

Fig. 1. Prone breast board.

Group breast contouring atlas. Tangential-fields treatment plan with 6-MV photons in each position was designed with prescription dose of 50 Gy in 2 Gy fractions, and then the dose-volume histograms for CTV and organs at risks were compared between prone and supine positions. Primary endpoint was the irradiated cardiac dose (heart and LADCA), and the secondary endpoints were the irradiated dose to ipsilateral lung and target coverage.

For target coverage, we compared the maximum and mean dose $\left(D_{\max }, D_{\text {mean }}\right)$, the dose received by at least $95 \%$ of the CTV $\left(\mathrm{D}_{95}\right)$, homogeneity index (HI) [25], and conformity index (CI) [26]. The HI was calculated from the formula: $\mathrm{HI}=\left(\mathrm{D}_{2}-\mathrm{D}_{98}\right) / \mathrm{D}_{\mathrm{p}}$, where $\mathrm{D}_{98}$ is the dose received by at least $98 \%$ of the target volume, $\mathrm{D}_{2}$ is the dose received by at least $2 \%$ of the target volume, and $D_{p}$ is the prescription dose to the target volume. The $\mathrm{D}_{98}$ and $\mathrm{D}_{2}$ are considered to be the minimum and maximum doses, respectively, and a lower $\mathrm{HI}$ value indicates a more homogeneous dose administered to the target volume. The $\mathrm{CI}$ was calculated from the formula: $\mathrm{CI}=\left(\mathrm{TV}_{95} / \mathrm{TV}\right) \times\left(\mathrm{TV}_{95} / \mathrm{V}_{95}\right)$, where $\mathrm{V}_{95}$ is the target volume receiving $95 \%$ of the prescription dose, TV is the target volume, and $\mathrm{V}_{95}$ is the volume receiving $95 \%$ of the prescription dose. The closer the $\mathrm{CI}$ is to 1 is indicative of optimal conformation. For normal organs, we compared $D_{\max }, D_{\text {mean, }}$ and the percentage of the volume that receives more than $5,10,20$, 30 , 40, and $50 \mathrm{~Gy}\left(\mathrm{~V}_{5}, \mathrm{~V}_{10}, \mathrm{~V}_{20}, \mathrm{~V}_{30}, \mathrm{~V}_{40}\right.$, and $\left.\mathrm{V}_{50}\right)$.

\section{Treatment position designation}

According to study protocol, the treatment position was designated to deliver less radiation dose to the heart and LADCA, considering the maximum dose and irradiated volume, while maintaining the appropriate dose $(95 \%-107 \%)$ of the prescribed dose to CTV. The LADCA dose was considered to be the determining factor of the highest priority.

As a process of designation, we evaluated the parameters between prone- and supine-position-beneficial groups for each position. Age and chest and bust sizes, volumes of CTV, heart, and LADCA in both prone and supine positions were comparable between the two groups. Lung volume in prone position for prone-position-beneficial group was smaller than for supine-position-beneficial group $(1,162.1 \pm 201.1 \mathrm{~mL}$ vs. $1,296.4 \pm 224.7 \mathrm{~mL}, \mathrm{p}=0.035)$, whereas lung volume in supine position between the two groups was not statistically different $(1,081.4 \pm 213.7 \mathrm{~mL}$ vs. $1,197.1 \pm 232.7 \mathrm{~mL}, \mathrm{p}=0.081)$. Target dose parameters for each position were all comparable between the two groups. Lung, heart, and LADCA dose in prone position was significantly lower for prone-positionbeneficial group. In supine position, the lung dose was comparable between the two groups but heart and LADCA dose was significantly lower for supine-position-beneficial group than for prone-position-beneficial group.

\section{Treatment and verification}

The treatment was performed using a Varian Clinac 6Ex machine (Varian Medical Systems, Palo Alto, CA). For the patient setup verification, a weekly portal image using an electronic portal imaging device (EPID) was obtained. Intrafractional motion was assessed with images acquired at a rate of two images per second using an EPID in cine mode during one of the tangential beam delivery. To assess quantitatively, an external marker was placed on the left breast tattoo during imaging time. The displacement of marker was compared in portal images with that of the first fraction for interfractional setup verification, and the maximum movement of marker during beam delivery was analyzed in the cine images for intrafractional motion.

\section{Statistical analysis}

Dosimetric parameters were examined by paired $t$ test or Wilcoxon signed-rank test. The correlation between dosimetric benefit and treatment position were analyzed by paired $\mathrm{t}$ test or non-parametric statistical test including KruskalWallis test and Mann-Whitney U test. Inter-/intra-fractional 
motions of patients were evaluated by independent samples $\mathrm{t}$ test. Statistical analyses were conducted using SPSS ver. 20.0.0 (IBM Corp., Armonk, NY). We considered a p-value of less than 0.05 to be statistically significant.

\section{Ethical statement}

This prospective study received approval from Institutional Review Board of Samsung Medical Center (IRB No. 2014-06-138-002) and performed in accordance with the principles of the Declaration of Helsinki and Good Clinical Practice guidelines. All patients provided written informed consent before enrollment in this study. The trial was registered at ClinicalTrials.gov (NCT02231112).

\section{Results}

\section{Total 50 enrolled patients}

The characteristics of total 50 enrolled patients are listed in Table 1. The median age of total 50 patients was 48 years (range, 36 to 64 years). The median chest size was $78 \mathrm{~cm}$ (range, 60 to $90 \mathrm{~cm}$ ) and the median bust size was $88 \mathrm{~cm}$ (range, 72 to $107 \mathrm{~cm}$ ). The median breast, heart, and lung volumes measured in supine position was $269.3 \mathrm{~mL}$ (range, 95.3 to $605.6 \mathrm{~mL}$ ), $595.7 \mathrm{~mL}$ (range, 455.1 to $800.1 \mathrm{~mL}$ ), and 1,137.9 $\mathrm{mL}(701.2-1,843.9 \mathrm{~mL})$, respectively.

\section{Dosimetric beneficial group}

We performed the comparisons between prone and supine positions for the entire patients and the comparisons of parameters are summarized in Table 2. Prone position significantly increased the average volume of breast (CTV) (304.8 $\pm 149.3 \mathrm{~mL}$ vs. $285.0 \pm 126.5 \mathrm{~mL}, \mathrm{p}=0.001)$. Ipsilateral lung volume was also significantly increased while heart volume was decreased in prone position $(1,218.5 \pm 219.6 \mathrm{~mL}$ vs. $1,130 \pm$ $227 \mathrm{~mL}, \mathrm{p}<0.001$ and $590.9 \pm 85.7 \mathrm{~mL}$ vs. $614.0 \pm 91.7 \mathrm{~mL}$, $\mathrm{p}<0.001$, respectively). The volume of LADCA was equivalent in both positions. The radiation dose to the CTV was similar when comparing $\mathrm{D}_{\max }$ and $\mathrm{D}_{95}$ of prone and supine positions. The $\mathrm{D}_{\text {mean }}$ of CTV was statistically larger in prone position but the absolute difference of the mean value between two positions was only $0.3 \mathrm{~Gy}(50.8 \pm 0.4 \mathrm{~Gy}$ vs. $50.5 \pm 0.5$ $\mathrm{Gy}, \mathrm{p}<0.001)$. The $\mathrm{CI}$ in prone position was closer to 1 indicating more optimal target conformation $(0.97 \pm 0.05$ vs $0.86 \pm$ $0.14, \mathrm{p}<0.001)$ while the HI was similar in both positions. All dose values ( $D_{\max }, D_{\text {mean, }}$ and $V_{5}-V_{50}$ ) of ipsilateral lung, heart, and LADCA were lower in prone position than in
Table 1. Characteristics of total 50 participants

\begin{tabular}{|c|c|}
\hline Characteristic & No. $(\%)$ \\
\hline Age (yr) & $48(36-64)$ \\
\hline \multicolumn{2}{|l|}{ Type of breast cancer } \\
\hline Invasive ductal carcinoma & $40(80)$ \\
\hline Ductal carcinoma in situ & $3(6)$ \\
\hline Mixed invasive ductal carcinoma & $3(6)$ \\
\hline Others & $4(8)$ \\
\hline \multicolumn{2}{|l|}{ Surgery } \\
\hline Partial mastectomy & $2(4)$ \\
\hline $\begin{array}{l}\text { Partial mastectomy+ } \\
\text { sentinel lymph node dissection }\end{array}$ & $48(96)$ \\
\hline \multicolumn{2}{|l|}{ Pathologic T category } \\
\hline Tis & $3(6)$ \\
\hline T1 & $42(84)$ \\
\hline $\mathrm{T} 2$ & $5(10)$ \\
\hline \multicolumn{2}{|l|}{ Pathologic N category } \\
\hline N0 & $49(98)$ \\
\hline $\mathrm{Nx}$ & $1(2)$ \\
\hline Chest size (cm) & $78(60-90)$ \\
\hline Bust size $(\mathrm{cm})$ & $88(72-107)$ \\
\hline Breast volume $^{\mathrm{a})}(\mathrm{mL})$ & $269.3(95.3-605.6)$ \\
\hline Heart volume $\left.{ }^{a}\right)(m L)$ & $595.7(455.1-800.1)$ \\
\hline Lung volume $\left.{ }^{a}\right)(m L)$ & $1,137.9(701.2-1,843.9)$ \\
\hline
\end{tabular}

supine position (Table 2).

Among these 50 patients, 32 cases were decided as proneposition-beneficial group and 18 cases as supine-positionbeneficial group in terms of dosimetric advantage after the dosimetric results of treatment plans for both setups were thoroughly compared according to study protocol. The comparisons between prone and supine positions for each proneand supine-position-beneficial group are described in Table 3. Age, chest size, and bust size between prone-position-beneficial and supine-position-beneficial groups were comparable $(p=0.723, p=0.356$, and $p=0.550$, respectively). For proneposition-beneficial group, prone position significantly increased lung volume $(1,162.1 \pm 201.1 \mathrm{~mL}$ vs. $1,081.4 \pm 213.7$ $\mathrm{mL}, \mathrm{p}<0.001)$ but decreased heart volume $(591.7 \pm 87.9 \mathrm{~mL}$ vs. $624.6 \pm 96.5 \mathrm{~mL}, \mathrm{p}<0.001)$, while CTV was not statistically different between the two positions. In contrast, for supineposition-beneficial group, prone position also increased lung volume $(1,296.4 \pm 224.7 \mathrm{~mL}$ vs. $1,197.1 \pm 232.7 \mathrm{~mL}, \mathrm{p}=0.002)$ as well as CTV (323.0 $\pm 182.0 \mathrm{~mL}$ vs. $285.1 \pm 138.6 \mathrm{~mL}, \mathrm{p}=0.004)$, but heart volume was comparable between two setups (589.6 $\pm 84.7 \mathrm{~mL}$ vs. $599.3 \pm 84.6 \mathrm{~mL}, \mathrm{p}=0.200)$. LADCA volume was comparable between the two setups for both groups. 
Table 2. Comparison between prone and supine positions for total 50 patients

\begin{tabular}{|c|c|c|c|}
\hline \multirow{2}{*}{ Variable } & \multicolumn{3}{|c|}{ Total patients $(n=50)$} \\
\hline & Prone position & Supine position & p-value \\
\hline Age (yr) & \multicolumn{2}{|c|}{$49 \pm 7$} & \\
\hline Chest size $(\mathrm{cm})$ & \multicolumn{2}{|c|}{$78.4 \pm 6.3$} & \\
\hline Bust size (cm) & \multicolumn{2}{|c|}{$88.3 \pm 7.5$} & \\
\hline \multicolumn{4}{|l|}{ Volume (mL) } \\
\hline CTV (breast) & $304.8 \pm 149.3$ & $285.0 \pm 126.5$ & 0.001 \\
\hline Lung & $1,218.5 \pm 219.6$ & $1,130.0 \pm 227.0$ & $<0.001$ \\
\hline Heart & $590.9 \pm 85.7$ & $614.0 \pm 91.7$ & $<0.001$ \\
\hline LADCA & $0.9 \pm 0.2$ & $0.9 \pm 0.2$ & 0.039 \\
\hline \multicolumn{4}{|l|}{ Target dose } \\
\hline $\mathrm{D}_{\max }(\mathrm{Gy})$ & $53.1 \pm 0.4$ & $52.9 \pm 0.3$ & 0.076 \\
\hline $\mathrm{D}_{\text {mean }}(\mathrm{Gy})$ & $50.8 \pm 0.4$ & $50.5 \pm 0.5$ & $<0.001$ \\
\hline $\mathrm{D}_{95}(\mathrm{~Gy})$ & $48.9 \pm 0.6$ & $48.7 \pm 0.7$ & 0.053 \\
\hline $\mathrm{HI}$ & $0.09 \pm 0.02$ & $0.10 \pm 0.10$ & 0.463 \\
\hline $\mathrm{CI}$ & $0.97 \pm 0.05$ & $0.86 \pm 0.14$ & $<0.001$ \\
\hline \multicolumn{4}{|l|}{ Dose in OAR } \\
\hline \multicolumn{4}{|l|}{ Lung } \\
\hline $\mathrm{D}_{\max }(\mathrm{Gy})$ & $38.8 \pm 14.6$ & $50.8 \pm 1.2$ & $<0.001$ \\
\hline $\mathrm{D}_{\text {mean }}(\mathrm{Gy})$ & $1.4 \pm 1.0$ & $7.5 \pm 1.8$ & $<0.001$ \\
\hline $\mathrm{V}_{5}(\%)$ & $3.4 \pm 3.4$ & $23.2 \pm 4.5$ & $<0.001$ \\
\hline $\mathrm{V}_{10}(\%)$ & $2.2 \pm 2.5$ & $17.7 \pm 4.3$ & $<0.001$ \\
\hline $\mathrm{V}_{20}(\%)$ & $1.5 \pm 1.9$ & $14.0 \pm 4.1$ & $<0.001$ \\
\hline $\mathrm{V}_{30}(\%)$ & $1.1 \pm 1.6$ & $11.8 \pm 4.0$ & $<0.001$ \\
\hline $\mathrm{V}_{40}(\%)$ & $0.7 \pm 1.2$ & $8.8 \pm 3.6$ & $<0.001$ \\
\hline $\mathrm{V}_{50}(\%)$ & $0 \pm 0$ & $0.2 \pm 0.4$ & $<0.001$ \\
\hline \multicolumn{4}{|l|}{ Heart } \\
\hline $\mathrm{D}_{\max }(\mathrm{Gy})$ & $42.6 \pm 9.7$ & $48.2 \pm 6.8$ & 0.002 \\
\hline $\mathrm{D}_{\text {mean }}(\mathrm{Gy})$ & $2.4 \pm 1.2$ & $3.4 \pm 3.6$ & 0.063 \\
\hline $\mathrm{V}_{5}(\%)$ & $5.1 \pm 3.6$ & $6.8 \pm 3.5$ & 0.015 \\
\hline $\mathrm{V}_{10}(\%)$ & $3.3 \pm 2.9$ & $4.7 \pm 2.9$ & 0.014 \\
\hline $\mathrm{V}_{20}(\%)$ & $2.4 \pm 2.5$ & $3.6 \pm 2.4$ & 0.017 \\
\hline $\mathrm{V}_{30}(\%)$ & $1.9 \pm 2.2$ & $2.9 \pm 2.1$ & 0.019 \\
\hline $\mathrm{V}_{40}(\%)$ & $1.3 \pm 1.8$ & $2.2 \pm 1.8$ & 0.019 \\
\hline $\mathrm{V}_{50}(\%)$ & $0 \pm 0$ & $0.1 \pm 0.2$ & 0.005 \\
\hline \multicolumn{4}{|l|}{ LADCA } \\
\hline $\mathrm{D}_{\max }(\mathrm{Gy})$ & $30.9 \pm 15.8$ & $41.8 \pm 12.7$ & $<0.001$ \\
\hline $\mathrm{D}_{\text {mean }}(\mathrm{Gy})$ & $5.6 \pm 3.8$ & $10.0 \pm 5.8$ & $<0.001$ \\
\hline $\mathrm{V}_{5}(\%)$ & $17.7 \pm 9.1$ & $28.8 \pm 13.1$ & $<0.001$ \\
\hline $\mathrm{V}_{10}(\%)$ & $10.5 \pm 9.2$ & $22.0 \pm 13.1$ & $<0.001$ \\
\hline $\mathrm{V}_{20}(\%)$ & $8.0 \pm 8.9$ & $18.2 \pm 13.0$ & $<0.001$ \\
\hline $\mathrm{V}_{30}(\%)$ & $6.4 \pm 8.1$ & $15.9 \pm 12.5$ & $<0.001$ \\
\hline $\mathrm{V}_{40}(\%)$ & $5.0 \pm 7.6$ & $12.8 \pm 11.5$ & $<0.001$ \\
\hline $\mathrm{V}_{50}(\%)$ & $0 \pm 0$ & $0.6 \pm 2.8$ & 0.147 \\
\hline
\end{tabular}

Values are presented as mean \pm standard deviation. CTV, clinical target volume; LADCA, left anterior descending coronary artery; $\mathrm{D}_{\max }$, maximum dose; $\mathrm{D}_{\text {mean, }}$ mean dose; $\mathrm{D}_{95}$, dose received by at least $95 \%$ of the clinical target volume; HI, homogeneity index; $\mathrm{CI}$, conformity index; $\mathrm{OAR}$, organs at risk; $\mathrm{Vx}$, percentage of the volume that receives more than $\mathrm{X}$ Gy. 
Table 3. Comparison between prone and supine positions for prone- and supine-position-beneficial groups

\begin{tabular}{|c|c|c|c|c|c|c|}
\hline \multirow[b]{2}{*}{ Variable } & \multicolumn{3}{|c|}{ Prone-position-beneficial group $(n=32)$} & \multicolumn{3}{|c|}{ Supine-position-beneficial group(n=18) } \\
\hline & $\begin{array}{c}\text { Prone } \\
\text { position }\end{array}$ & $\begin{array}{l}\text { Supine } \\
\text { position }\end{array}$ & p-value & $\begin{array}{c}\text { Prone } \\
\text { position }\end{array}$ & $\begin{array}{l}\text { Supine } \\
\text { position }\end{array}$ & p-value \\
\hline Age (yr) & \multicolumn{2}{|c|}{$48 \pm 8$} & & \multicolumn{2}{|c|}{$49 \pm 8$} & \\
\hline Chest size $(\mathrm{cm})$ & \multicolumn{2}{|c|}{$77.9 \pm 6.1$} & & \multicolumn{2}{|c|}{$79.2 \pm 6.8$} & \\
\hline Bust size $(\mathrm{cm})$ & \multicolumn{2}{|c|}{$87.6 \pm 6.9$} & & \multicolumn{2}{|c|}{$89.6 \pm 8.5$} & \\
\hline \multicolumn{7}{|l|}{ Volume (mL) } \\
\hline CTV (Breast) & $291.6 \pm 122.2$ & $284.9 \pm 119.5$ & 0.102 & $323.0 \pm 182.0$ & $285.1 \pm 138.6$ & 0.004 \\
\hline Lung & $1,162.1 \pm 201.1$ & $1,081.4 \pm 213.7$ & $<0.001$ & $1,296.4 \pm 224.7$ & $1,197.1 \pm 232.7$ & 0.002 \\
\hline Heart & $591.7 \pm 87.9$ & $624.6 \pm 96.5$ & $<0.001$ & $589.6 \pm 84.7$ & $599.3 \pm 84.6$ & 0.200 \\
\hline LADCA & $1.0 \pm 0.2$ & $0.9 \pm 0.2$ & 0.056 & $0.9 \pm 0.2$ & $0.9 \pm 0.2$ & 0.451 \\
\hline \multicolumn{7}{|l|}{ Target dose } \\
\hline $\mathrm{D}_{\max }(\mathrm{Gy})$ & $53.1 \pm 0.5$ & $52.9 \pm 0.3$ & 0.019 & $53.0 \pm 0.3$ & $53.0 \pm 0.3$ & 0.732 \\
\hline $\mathrm{D}_{\text {mean }}(\mathrm{Gy})$ & $50.8 \pm 0.4$ & $50.5 \pm 0.4$ & 0.001 & $50.8 \pm 0.3$ & $50.5 \pm 0.5$ & 0.083 \\
\hline $\mathrm{D}_{95}(\mathrm{~Gy})$ & $49.0 \pm 0.6$ & $48.7 \pm 0.6$ & 0.099 & $48.8 \pm 0.6$ & $48.6 \pm 0.8$ & 0.323 \\
\hline $\mathrm{HI}$ & $0.08 \pm 0.02$ & $0.10 \pm 0.13$ & 0.379 & $0.09 \pm 0.02$ & $0.08 \pm 0.02$ & 0.219 \\
\hline CI & $0.99 \pm 0.02$ & $0.85 \pm 0.14$ & $<0.001$ & $0.96 \pm 0.07$ & $0.86 \pm 0.15$ & 0.002 \\
\hline \multicolumn{7}{|l|}{ Dose in OAR } \\
\hline \multicolumn{7}{|l|}{ Lung } \\
\hline $\mathrm{D}_{\max }(\mathrm{Gy})$ & $34.9 \pm 16$ & $51.0 \pm 1.1$ & $<0.001$ & $44.2 \pm 10.4$ & $50.5 \pm 1.2$ & 0.023 \\
\hline $\mathrm{D}_{\text {mean }}(\mathrm{Gy})$ & $0.9 \pm 0.6$ & $7.6 \pm 2.0$ & $<0.001$ & $1.9 \pm 1.2$ & $7.4 \pm 1.6$ & $<0.001$ \\
\hline $\mathrm{V}_{5}(\%)$ & $2.0 \pm 2.1$ & $23.1 \pm 4.9$ & $<0.001$ & $5.2 \pm 4$ & $23.4 \pm 4.0$ & $<0.001$ \\
\hline $\mathrm{V}_{10}(\%)$ & $1.2 \pm 1.4$ & $17.7 \pm 4.6$ & $<0.001$ & $3.5 \pm 2.9$ & $17.5 \pm 3.9$ & $<0.001$ \\
\hline $\mathrm{V}_{20}(\%)$ & $0.7 \pm 1$ & $14.2 \pm 4.4$ & $<0.001$ & $2.6 \pm 2.3$ & $13.7 \pm 3.7$ & $<0.001$ \\
\hline $\mathrm{V}_{30}(\%)$ & $0.5 \pm 0.8$ & $12.0 \pm 4.2$ & $<0.001$ & $2.0 \pm 1.9$ & $11.4 \pm 3.6$ & $<0.001$ \\
\hline $\mathrm{V}_{40}(\%)$ & $0.3 \pm 0.5$ & $9.2 \pm 3.9$ & $<0.001$ & $1.4 \pm 1.5$ & $8.3 \pm 3.2$ & $<0.001$ \\
\hline $\mathrm{V}_{50}(\%)$ & $0 \pm 0$ & $0.3 \pm 0.5$ & 0.002 & $0 \pm 0$ & $0.1 \pm 0.2$ & 0.019 \\
\hline \multicolumn{7}{|l|}{ Heart } \\
\hline $\mathrm{D}_{\max }(\mathrm{Gy})$ & $40.6 \pm 10.5$ & $49.8 \pm 1.2$ & $<0.001$ & $45.4 \pm 7.7$ & $45.9 \pm 10.1$ & 0.957 \\
\hline $\mathrm{D}_{\text {mean }}(\mathrm{Gy})$ & $2.0 \pm 0.8$ & $4.2 \pm 4.6$ & 0.016 & $2.9 \pm 1.5$ & $2.4 \pm 1.0$ & 0.016 \\
\hline $\mathrm{V}_{5}(\%)$ & $4.0 \pm 2.6$ & $8.1 \pm 3.2$ & $<0.001$ & $6.5 \pm 4.4$ & $4.9 \pm 3.1$ & 0.014 \\
\hline $\mathrm{V}_{10}(\%)$ & $2.5 \pm 2$ & $5.9 \pm 2.7$ & $<0.001$ & $4.5 \pm 3.6$ & $3.2 \pm 2.3$ & 0.022 \\
\hline $\mathrm{V}_{20}(\%)$ & $1.7 \pm 1.6$ & $4.6 \pm 2.4$ & $<0.001$ & $3.4 \pm 3.2$ & $2.3 \pm 1.9$ & 0.023 \\
\hline $\mathrm{V}_{30}(\%)$ & $1.3 \pm 1.3$ & $3.7 \pm 2.2$ & $<0.001$ & $2.7 \pm 2.8$ & $1.8 \pm 1.5$ & 0.027 \\
\hline $\mathrm{V}_{40}(\%)$ & $0.8 \pm 1$ & $2.8 \pm 1.9$ & $<0.001$ & $2.0 \pm 2.3$ & $1.3 \pm 1.2$ & 0.038 \\
\hline $\mathrm{V}_{50}(\%)$ & $0 \pm 0$ & $0.1 \pm 0.2$ & 0.017 & $0 \pm 0$ & $0 \pm 0.1$ & 0.083 \\
\hline \multicolumn{7}{|l|}{ LADCA } \\
\hline $\mathrm{D}_{\max }(\mathrm{Gy})$ & $29.3 \pm 14.6$ & $47.2 \pm 5.6$ & $<0.001$ & $33.0 \pm 17.5$ & $34.2 \pm 15.8$ & 0.349 \\
\hline $\mathrm{D}_{\text {mean }}(\mathrm{Gy})$ & $4.6 \pm 2.6$ & $12.8 \pm 5.5$ & $<0.001$ & $7.0 \pm 4.7$ & $6.3 \pm 3.8$ & 0.099 \\
\hline $\mathrm{V}_{5}(\%)$ & $16.5 \pm 7.4$ & $33.6 \pm 13.3$ & $<0.001$ & $19.4 \pm 10.9$ & $22.1 \pm 9.8$ & 0.588 \\
\hline $\mathrm{V}_{10}(\%)$ & $8.4 \pm 7.1$ & $27.9 \pm 12.4$ & $<0.001$ & $13.4 \pm 11$ & $14 \pm 9.3$ & 0.302 \\
\hline $\mathrm{V}_{20}(\%)$ & $5.8 \pm 6.8$ & $24.3 \pm 12.1$ & $<0.001$ & $11.1 \pm 10.7$ & $9.9 \pm 9.2$ & 0.037 \\
\hline $\mathrm{V}_{30}(\%)$ & $4.3 \pm 6.1$ & $21.7 \pm 11.5$ & $<0.001$ & $9.3 \pm 9.8$ & $7.7 \pm 8.9$ & 0.013 \\
\hline $\mathrm{V}_{40}(\%)$ & $2.9 \pm 5$ & $17.9 \pm 11.3$ & $<0.001$ & $7.9 \pm 9.5$ & $5.9 \pm 7.4$ & 0.008 \\
\hline $\mathrm{V}_{50}(\%)$ & $0 \pm 0$ & $1 \pm 3.7$ & 0.154 & $0 \pm 0$ & $0 \pm 0.1$ & 0.331 \\
\hline
\end{tabular}

Values are presented as mean \pm standard deviation. CTV, clinical target volume; LADCA, left anterior descending coronary artery; $\mathrm{D}_{\max }$, maximum dose; $\mathrm{D}_{\text {mean, }}$ mean dose; $\mathrm{D}_{95}$, dose received by at least $95 \%$ of the clinical target volume; HI, homogeneity index; $\mathrm{CI}$, conformity index; $\mathrm{OAR}$, organs at risk; $\mathrm{Vx}$, percentage of the volume that receives more than $\mathrm{X}$ Gy. 

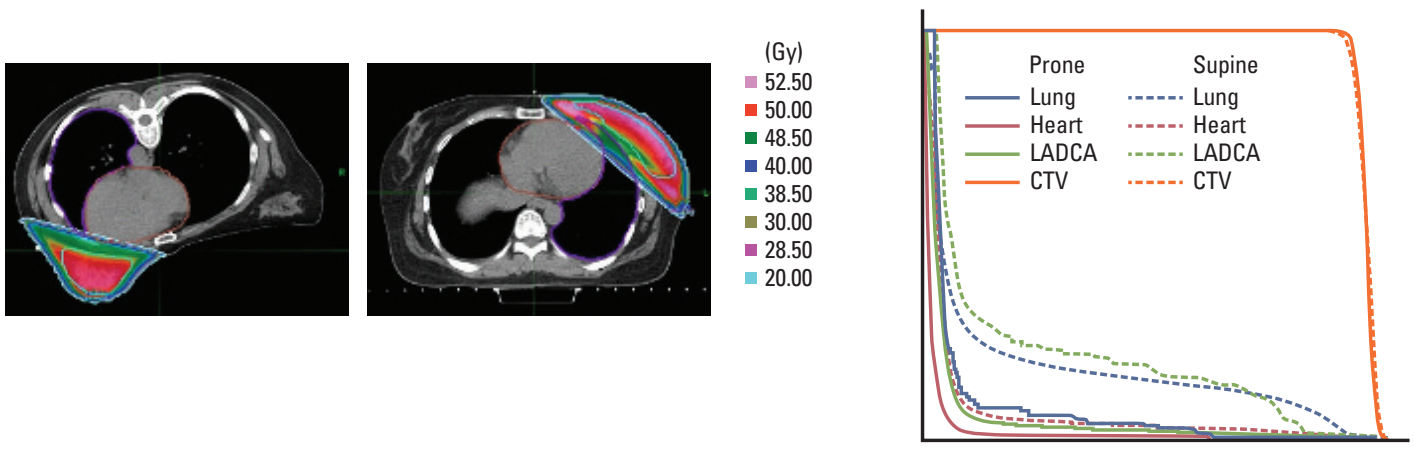

B
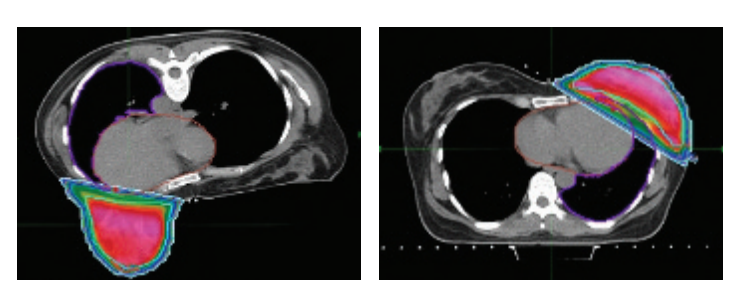

$\begin{aligned} & \text { (Gy) } \\ = & 52.50 \\ = & 50.00 \\ = & 48.50 \\ = & 40.00 \\ = & 38.50 \\ = & 30.00 \\ = & 28.50 \\ = & 20.00\end{aligned}$

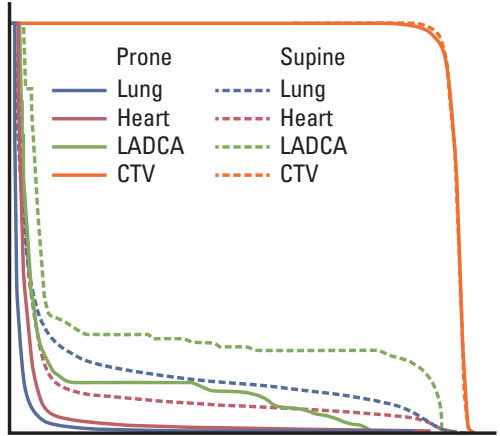

Fig. 2. Dosimetric comparison of organs at risk between supine and prone positioning with isodose lines and dose volume histogram for a case with chest size of $84 \mathrm{~cm}$ and breast volume in prone position of $302 \mathrm{~mL}$ (A) and a case with chest size of $68 \mathrm{~cm}$ and breast volume in prone position of $462 \mathrm{~mL}$ (B). The lung (blue), heart (red), and left anterior descending coronary artery (LADCA, green) are delineated as organs at risk. CTV, clinical target volume.

\section{Target coverage}

Target coverage dose was comparable between two setups for supine-position-beneficial group in terms of $\mathrm{D}_{\max }, \mathrm{D}_{\text {mean, }}$ and $D_{95}$. Target dose $\left(D_{\max }\right.$ and $\left.D_{\text {mean }}\right)$ was higher in prone position than in supine position for prone-position-beneficial group, but the absolute differences of those values were very minimal (Table 3). For both groups, HI was comparable between prone and supine positions, but CI was more optimal in prone position than in supine position (prone-positionbeneficial group, $0.99 \pm 0.02$ vs. $0.85 \pm 0.14$, $\mathrm{p}<0.001$; supineposition-beneficial group, $0.96 \pm 0.07$ vs. $0.86 \pm 0.15, p=0.002$ ) (Table 3).

\section{Normal organ sparing}

Lung dose $\left(D_{\max }, D_{\text {mean, }}\right.$ and $\left.V_{5}-V_{50}\right)$ was substantially decreased in prone position than in supine position for both prone and supine-position-beneficial groups, along with the fact that absolute dose differences were considerably larger in prone-position-beneficial group than in supine-position- beneficial group (Table 3). Prone position for prone-positionbeneficial group significantly decreased the dose to heart and LADCA $\left(D_{\max }, D_{\text {mean, }}\right.$ and $\left.V_{5}-V_{50}\right)$. On the other hand, prone position for supine-position-beneficial group significantly increased the heart dose $\left(D_{\text {mean }}\right.$ and $\left.V_{5}-V_{50}\right)$ (Table 3$)$. For supine-position-beneficial group, prone position increased $\mathrm{V}_{20}, \mathrm{~V}_{30}$, and $\mathrm{V}_{40}$ of LADCA considerably, while $\mathrm{D}_{\max }, \mathrm{D}_{\text {mean, }}$ $\mathrm{V}_{5}, \mathrm{~V}_{10}$, and $\mathrm{V}_{50}$ of LADCA were similar (Table 3). Fig. 2 showed dosimetric comparison of normal organs between supine and prone position with isodose lines and dose volume histogram for a case with chest size of $84 \mathrm{~cm}$ and breast volume in prone position of $302 \mathrm{~mL}$ (Fig. 2A) and a case with chest size of $68 \mathrm{~cm}$ and breast volume in prone position of $462 \mathrm{~mL}$ (Fig. 2B).

\section{Patient motion}

Comparisons of inter- and intra-fractional motions between patients treated in supine and prone positions are depicted in Fig. 3. For interfractional movement, a total of 116 EPID images of prone position treatments and 74 images 
A

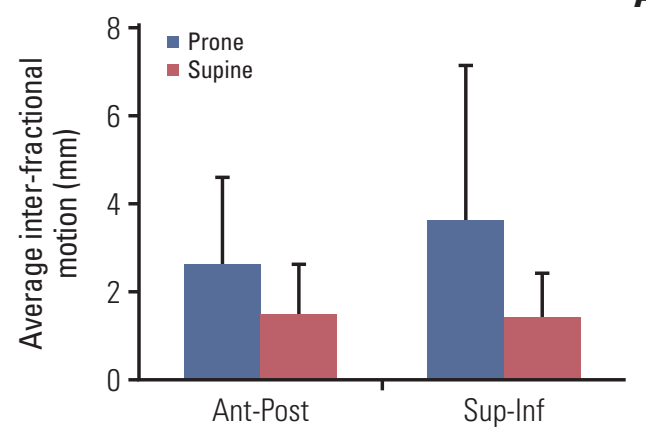

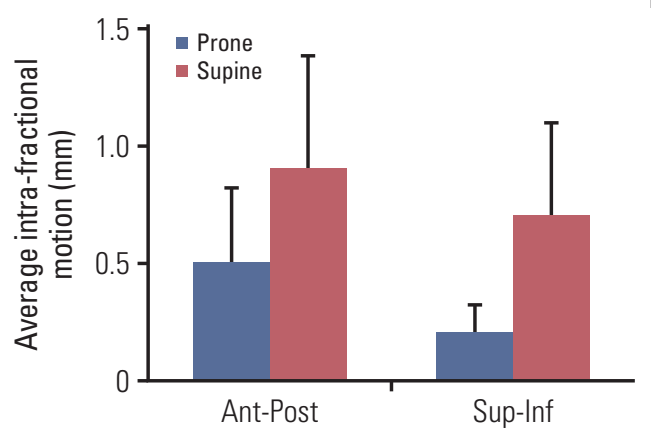

Fig. 3. Comparison of inter-(A) and intra-fractional (B) motion between patients treated in supine and prone positions. AntPost, anterior-posterior; Sup-Inf, superior-inferior.

of supine position treatments were reviewed. The average of setup variation was $3.57 \pm 3.56 \mathrm{~mm}$ in superior-inferior (SI) direction and $2.61 \pm 2.02 \mathrm{~mm}$ in anterior-posterior (AP) direction for prone position treatment and $1.35 \pm 1.05 \mathrm{~mm}$ in SI direction and $1.46 \pm 1.14 \mathrm{~mm}$ in AP direction for supine position treatment. Intrafractional motion was assessed by $0.19 \pm 0.13 \mathrm{~mm}$ in SI direction and $0.48 \pm 0.32 \mathrm{~mm}$ in AP direction from 140 EPID cine images of prone position treatment and $0.72 \pm 0.40 \mathrm{~mm}$ in SI direction and $0.86 \pm 0.49 \mathrm{~mm}$ in AP direction from 99 EPID images of supine position treatment. Interfractional setup variation was larger in prone position treatment ( $p<0.001$ for both SI and AP direction), whereas motion during treatment was lager in supine position treatment ( $\mathrm{p}<0.001$ for both SI and AP direction).

\section{Discussion}

To save the heart for left breast cancer patients, several techniques that displace the heart from the irradiation field have been utilized, which include respiratory motion management (Breath hold) or patient positioning in prone or lateral decubitus. Breath hold technique usually requires more than twice as much treatment time as conventional RT, and patients must control their own breathing, which is not feasible in elderly patients or those with poor performance status [14]. In case of lateral decubitus position, due to relatively unstable posture, the reproducibility, which is one of the most important challenges in RT, may be seriously degraded. Even though the prone position may also be discomfort during the treatment compared to the supine position, it is more advantageous than the two methods mentioned above [13].

Prone breast RT has been known as effective for patients with large pendulous breast, such as breast volume larger than $750 \mathrm{~mL}$ or $1,000 \mathrm{~mL}[19,20]$. As Asian women usually have a smaller breast size, prone position was considered not having dosimetric advantage to Asian breast cancer patients. There were studies about the breast size, but they have not involved such small breasts [22] or prone position did not reduce in-field heart volume statistically significantly in women with breast size smaller than $750 \mathrm{~mL}$ [17]. Even in Asian studies, patients with relatively large breast size were evaluated for prone positioning $[23,27]$. However, this prospective study enrolled by 50 patients with mean breast size of $305 \mathrm{~mL}$ (in prone position) revealed that such small breast size could also gain dosimetric benefit on heart and lung by prone position while sustaining or even improving target coverage.

CTV breast volume of total 50 participants ranged 94.2 to $786.5 \mathrm{~mL}$ (median, $294.1 \mathrm{~mL}$ ) in prone position and 95.3 to $605.6 \mathrm{~mL}$ (median, $269.3 \mathrm{~mL}$ ) in supine position. Prone position significantly increased the average CTV volume (304.8 $149.3 \mathrm{~mL}$ vs. $285.0 \pm 126.5 \mathrm{~mL}, \mathrm{p}=0.001$ ) in total patients, but the average CTV volume was comparable between prone and supine-position-beneficial groups (prone 291.6 \pm 122.2 $\mathrm{mL}$ vs. supine $323.0 \pm 182.0 \mathrm{~mL}, \mathrm{p}=0.470$ ). Prone-position-beneficial group had CTV volume in prone position ranging from 103.2 to $638.4 \mathrm{~mL}$. Prone position increased the volume of ipsilateral lung, and the relative lung volume change by prone position was similar between prone and supine-position-beneficial groups. Yet, the heart volume decreased in prone position for total patients, but the relative heart volume change by prone position was greater in prone-positionbeneficial group than in supine-position-beneficial group (but not statistically significant). However, we could not find the relationship between dosimetric gain and patient characteristics (breast or chest size) or volume change by prone positioning. The certain optimal subgroup having definite benefit by prone position was difficult to find probably due to a small number of participant in this Phase II study and 
small range of patients' breast size. Nevertheless, we could find dosimetric advantage in prone breast RT for patients having such small breast size even around $100 \mathrm{ml}$. A further study with a larger number of patients will be needed to find the ideal patient group who will have definite dosimetric gain. This further study will allow us to identify the patient for prone breast RT without two CT simulations for the comparison between supine and prone positions so as to avoid unnecessary radiation exposure to the patient.

Similar to earlier studies about prone breast RT [28,29], our study also found that the treatment in prone position showed larger interfractional motion but smaller intrafractional motion than the treatment in supine position. The patient's breathing was restricted due to the compression of the chest wall by lying on the prone breast board, so the intrafractional motion was limited than the supine position. However, there is no correlation between patient characteristics and the interor intra-fractional motion. Among 32 patients having dosimetric benefit in prone position, three patients showed extremely unstable setup during the process for the localization of treatment center before starting treatment, so physicians reviewed their treatment plans whether there was a critical clinical decision point and decided to treat them in supine position. Due to the lack of resource in our institution, we could utilize only EPID not cone-beam CT for these treatments. So, we were able to check the patient setup in SI and AP directions but not in lateral direction. The lateral setup of the patient was confirmed by localizing laser and patient tat- too and by checking the position of a marker attached to the midline of the patient. We found that a few patients treated in prone position, who enrolled in early-phase of this study, experienced contralateral breast exposure, especially with collimator rotation in the treatment parameter. So, we checked whether radiation fields extended to the contralateral breast with Gafchromic EBT3 film placed under the contralateral breast of the patient in the first few sessions of treatment. Also, we tried not to rotate the collimator in the treatment plan for prone position. With these efforts, the contralateral breast exposure problem was resolved.

In conclusion, prone breast RT could be beneficial to a subset of small breast patients since it substantially spared normal organs while achieving adequate coverage of breast tissue. We observed patient-specific and considerable interfractional setup error, but it was not the extent to negatively affect treatment quality of whole breast irradiation. Further prospective study is required to validate the potential benefit and the optimal patient group with prone breast RT.

\section{Conflicts of Interest}

Conflict of interest relevant to this article was not reported.

\section{Acknowledgments}

This research has been supported by Korean Breast Cancer Foundation 14-03.

\section{References}

1. Torre LA, Islami F, Siegel RL, Ward EM, Jemal A. Global cancer in women: burden and trends. Cancer Epidemiol Biomarkers Prev. 2017;26:444-57.

2. Jung KW, Won YJ, Kong HJ, Lee ES; Community of Population-Based Regional Cancer Registries. Cancer statistics in Korea: incidence, mortality, survival, and prevalence in 2015. Cancer Res Treat. 2018;50:303-16.

3. Early Breast Cancer Trialists' Collaborative Group; Darby S, McGale P, Correa C, Taylor C, Arriagada R, et al. Effect of radiotherapy after breast-conserving surgery on 10 -year recurrence and 15-year breast cancer death: meta-analysis of individual patient data for 10,801 women in 17 randomised trials. Lancet. 2011;378:1707-16.

4. Darby SC, McGale P, Taylor CW, Peto R. Long-term mortality from heart disease and lung cancer after radiotherapy for early breast cancer: prospective cohort study of about 300,000 women in US SEER cancer registries. Lancet Oncol. 2005;6:55765.

5. Marks LB, Yu X, Prosnitz RG, Zhou SM, Hardenbergh PH,
Blazing $\mathrm{M}$, et al. The incidence and functional consequences of RT-associated cardiac perfusion defects. Int J Radiat Oncol Biol Phys. 2005;63:214-23.

6. Correa CR, Litt HI, Hwang WT, Ferrari VA, Solin LJ, Harris EE. Coronary artery findings after left-sided compared with right-sided radiation treatment for early-stage breast cancer. J Clin Oncol. 2007;25:3031-7.

7. Darby SC, Ewertz M, McGale P, Bennet AM, Blom-Goldman $\mathrm{U}$, Bronnum D, et al. Risk of ischemic heart disease in women after radiotherapy for breast cancer. N Engl J Med. 2013;368: 987-98.

8. Paul Wright G, Drinane JJ, Sobel HL, Chung MH. Left-sided breast irradiation does not result in increased long-term cardiac-related mortality among women treated with breast-conserving surgery. Ann Surg Oncol. 2016;23:1117-22.

9. Yu TK, Whitman GJ, Thames HD, Buzdar AU, Strom EA, Perkins GH, et al. Clinically relevant pneumonitis after sequential paclitaxel-based chemotherapy and radiotherapy in breast cancer patients. J Natl Cancer Inst. 2004;96:1676-81. 
10. Borst GR, De Jaeger K, Belderbos JS, Burgers SA, Lebesque JV. Pulmonary function changes after radiotherapy in non-smallcell lung cancer patients with long-term disease-free survival. Int J Radiat Oncol Biol Phys. 2005;62:639-44.

11. Campana F, Kirova YM, Rosenwald JC, Dendale R, Vilcoq JR, Dreyfus $\mathrm{H}$, et al. Breast radiotherapy in the lateral decubitus position: a technique to prevent lung and heart irradiation. Int J Radiat Oncol Biol Phys. 2005;61:1348-54.

12. Kirova YM, Hijal T, Campana F, Fournier-Bidoz N, Stilhart A, Dendale $\mathrm{R}$, et al. Whole breast radiotherapy in the lateral decubitus position: a dosimetric and clinical solution to decrease the doses to the organs at risk (OAR). Radiother Oncol. 2014;110:477-81.

13. Huppert N, Jozsef G, Dewyngaert K, Formenti SC. The role of a prone setup in breast radiation therapy. Front Oncol. 2011; 1:31.

14. Remouchamps VM, Letts N, Vicini FA, Sharpe MB, Kestin LL, Chen PY, et al. Initial clinical experience with moderate deepinspiration breath hold using an active breathing control device in the treatment of patients with left-sided breast cancer using external beam radiation therapy. Int J Radiat Oncol Biol Phys. 2003;56:704-15.

15. Shah C, Badiyan S, Berry S, Khan AJ, Goyal S, Schulte K, et al. Cardiac dose sparing and avoidance techniques in breast cancer radiotherapy. Radiother Oncol. 2014;112:9-16.

16. Mulliez T, Veldeman L, van Greveling A, Speleers B, Sadeghi S, Berwouts D, et al. Hypofractionated whole breast irradiation for patients with large breasts: a randomized trial comparing prone and supine positions. Radiother Oncol. 2013;108: 203-8.

17. Formenti SC, DeWyngaert JK, Jozsef G, Goldberg JD. Prone vs supine positioning for breast cancer radiotherapy. JAMA. 2012;308:861-3.

18. Lymberis SC, deWyngaert JK, Parhar P, Chhabra AM, FentonKerimian M, Chang J, et al. Prospective assessment of optimal individual position (prone versus supine) for breast radiotherapy: volumetric and dosimetric correlations in 100 patients. Int J Radiat Oncol Biol Phys. 2012;84:902-9.

19. Kirby AM, Evans PM, Donovan EM, Convery HM, Haviland JS, Yarnold JR. Prone versus supine positioning for whole and partial-breast radiotherapy: a comparison of non-target tissue dosimetry. Radiother Oncol. 2010;96:178-84.

20. Fernandez-Lizarbe E, Montero A, Polo A, Hernanz R, Moris $\mathrm{R}$, Formenti S, et al. Pilot study of feasibility and dosimetric comparison of prone versus supine breast radiotherapy. Clin Transl Oncol. 2013;15:450-9.

21. Bartlett FR, Colgan RM, Donovan EM, McNair HA, Carr K, Evans PM, et al. The UK HeartSpare Study (Stage IB): randomised comparison of a voluntary breath-hold technique and prone radiotherapy after breast conserving surgery. Radiother Oncol. 2015;114:66-72.

22. Hannan R, Thompson RF, Chen Y, Bernstein K, Kabarriti R, Skinner $\mathrm{W}$, et al. Hypofractionated whole-breast radiation therapy: does breast size matter? Int J Radiat Oncol Biol Phys. 2012;84:894-901.

23. Chen JL, Cheng JC, Kuo SH, Chan HM, Huang YS, Chen YH. Prone breast forward intensity-modulated radiotherapy for Asian women with early left breast cancer: factors for cardiac sparing and clinical outcomes. J Radiat Res. 2013;54:899-908.

24. Lee HY, Chang JS, Lee IJ, Park K, Kim YB, Suh CO, et al. The deep inspiration breath hold technique using Abches reduces cardiac dose in patients undergoing left-sided breast irradiation. Radiat Oncol J. 2013;31:239-46.

25. Yoon M, Park SY, Shin D, Lee SB, Pyo HR, Kim DY, et al. A new homogeneity index based on statistical analysis of the dose-volume histogram. J Appl Clin Med Phys. 2007;8:9-17.

26. Feuvret L, Noel G, Mazeron JJ, Bey P. Conformity index: a review. Int J Radiat Oncol Biol Phys. 2006;64:333-42.

27. Takahashi K, Morota M, Kagami Y, Okamoto H, Sekii S, Inaba $\mathrm{K}$, et al. Prospective study of postoperative whole breast radiotherapy for Japanese large-breasted women: a clinical and dosimetric comparisons between supine and prone positions and a dose measurement using a breast phantom. BMC Cancer. 2016;16:757.

28. Morrow NV, Stepaniak C, White J, Wilson JF, Li XA. Intra- and interfractional variations for prone breast irradiation: an indication for image-guided radiotherapy. Int J Radiat Oncol Biol Phys. 2007;69:910-7.

29. Mitchell J, Formenti SC, DeWyngaert JK. Interfraction and intrafraction setup variability for prone breast radiation therapy. Int J Radiat Oncol Biol Phys. 2010;76:1571-7. 